\title{
ANALYSIS OF FULLY DEVELOPED TURBULENT FLOW IN AN ECCENTRIC ANNULUS
}

\author{
HiROMOTO USUI AND KeNJi TSURUTA \\ Department of Chemical Engineering, Yamaguchi University, \\ Ube 755
}

\begin{abstract}
An analysis is presented of fully developed turbulent flow in an eccentric annulus. An integral transformation technique (Kirchhoff transformation) is applied to eccentric annuli in a wide range of eccentricity and radius ratio. Velocity distributions, wall shear stress distributions and friction factors are calculated and compared with the literature. Although the analysis cannot predict the secondary flow in an eccentric annulus, the calculated results show good agreement with the experimental results in the literature.
\end{abstract}

\section{Introduction}

In recent years, heat transfer problems in noncircular ducts have become important. Tube misalignment in a close-packed tubular heat exchanger often causes serious damage to heat transfer equipment. In the case of nuclear reactors, in particular, it has been pointed out that misalignment of a fuel rod caused by thermal stress finally leads to the local meltdown of the fuel rod. The flow in an annulus is useful as a model for longitudinal flow in a tube bundle. The present investigation attempts to simulate the fully developed turbulent flow in an eccentric annulus and to compare the predicted results with the available experimental results.

Some analytical methods for an eccentric annular flow have been developed, in the laminar region by Snyder and Goldstein ${ }^{9)}$ and in the turbulent region by Deissler and Taylor ${ }^{2}$. Deissler's method has wide applicability to an arbitrary shape of non-circular cross-sectional flow. However, the conformal mapping technique employed by Deissler is so tedious that a simpler method for the prediction of non-circular duct flow has been expected. Krajewski ${ }^{6}$ ) modified Deissler's method and applied it to a fully developed triangular duct flow. Recently, Launder and Ying ${ }^{7}$, and Aly et al. ${ }^{11}$ developed one equation turbulence model in square and triangular duct flows. This new method has the advantage of predicting the secondary flow in a non-circular duct flow. But the physical meaning of turbulence scale modeling is not clear, and more study seems to be needed. Thus, in this investigation, Krajewski's method is applied to an

Received May 6, 1980. Correspondence concerning this article should be addressed to H. Usui. K. Tsuruta is now with Obara Plating Industry Co. Ltd., Hiroshima 734. eccentric annulus.

Although an extremely large number of papers on concentric annuli have been published in recent years, papers concerning an eccentric annulus are rather scarce. Only the reports by Jonsson and Sparrow ${ }^{5}$, Dodge $^{3)}$ and Mizushina ${ }^{87}$ are available. Comparison will be made of these experimental results with the prediction of this work.

\section{Analysis}

\section{1 Laminar flow}

It is necessary to use the result of the laminar velocity profile in the analysis of turbulent flow, as will be shown in the following section. The analysis of laminar eccentric annular flows has been investigated by Snyder and Goldstein ${ }^{9}$. Their results are cited here briefly for the convenience of the following turbulence analysis. The geometry considered is shown in Fig. 1. The equation of motion for fully developed laminar flow may be given by

$$
\frac{\partial^{2} u}{\partial x^{2}}+\frac{\partial^{2} u}{\partial y^{2}}=\frac{1}{\mu} \frac{d p}{d z}
$$

The boundary conditions are $u=0$ at the inner and outer wall. Due to the asymmetry of the geometry,

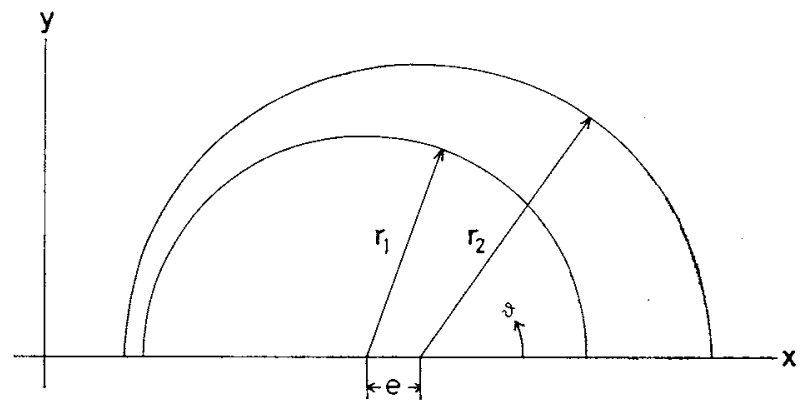

Fig. 1 Coordinate system 
bipolar coordinates are used in this study. The bipolar coordinates $(n, \xi)$ are defined by the transformation

$$
x+i y=i c \cot \left(\frac{\xi+i \eta}{2}\right)
$$

where $c$ is a constant and $i=\sqrt{-1}$. The inner and outer surface of the annulus are represented by lines of constant $\eta$ of which values will be designated as $\alpha$ and $\beta$, respectively. It may be shown from geometrical considerations that the constant $c, \alpha$ and $\beta$ are given by the expressions

$$
\begin{gathered}
c=r_{1} \sinh \alpha=r_{2} \sinh \beta \\
\cosh \alpha=\frac{1}{\gamma} \frac{\gamma\left(1+\phi^{2}\right)+\left(1-\phi^{2}\right)}{2 \phi} \\
\cosh \beta=\frac{\gamma\left(1-\phi^{2}\right)+\left(1+\phi^{2}\right)}{2 \phi}
\end{gathered}
$$

where

$$
\begin{aligned}
& \gamma=r_{1} / r_{2} \\
& \phi=e /\left(r_{2}-r_{1}\right)
\end{aligned}
$$

Transformation of Eq. (1) into bipolar coordinates gives

$$
\frac{\partial^{2} v}{\partial \xi^{2}}+\frac{\partial^{2} v}{\partial \eta^{2}}=-\frac{1}{(\cosh \eta-\cos \xi)^{2}}
$$

where

$$
v=-u /\left(\frac{c^{2}}{\mu} \frac{d p}{d z}\right)
$$

The general solution of Eq. (4a) is

$$
\begin{aligned}
v= & F+E \eta-\frac{\operatorname{ctnh} \eta}{2} \\
& +\sum_{n=1}^{\infty}\left\{A_{n} e^{n \eta}+\left(B_{n}-\operatorname{ctnh} \eta\right) e^{-n \eta} \cos n \xi\right\}
\end{aligned}
$$

where

$$
\begin{aligned}
F & =\frac{\alpha \operatorname{ctnh} \beta-\beta \operatorname{ctnh} \alpha}{2(\alpha-\beta)} \\
E & =\frac{\operatorname{ctnh} \alpha-\operatorname{ctnh} \beta}{2(\alpha-\beta)} \\
A_{n} & =\frac{\operatorname{ctnh} \alpha-\operatorname{ctnh} \beta}{e^{2 n \alpha}-e^{2 n \beta}} \\
B_{n} & =\frac{e^{2 n \alpha} \operatorname{ctnh} \beta-e^{2 n \beta} \operatorname{ctnh} \alpha}{e^{2 n \alpha}-e^{2 n \beta}}
\end{aligned}
$$

\section{2 Turbulent flow}

The equation of motion for fully developed turbulent flow in an eccentric annulus may be written as

$$
\frac{\partial}{\partial x}\left(1+\frac{\varepsilon_{m}}{\nu}\right) \frac{\partial u}{\partial x}+\frac{\partial}{\partial y}\left(1+\frac{\varepsilon_{m}}{\nu}\right) \frac{\partial u}{\partial y}=\frac{1}{\mu} \frac{d p}{d z}
$$

An integral transformation (Kirchhoff transformation) defined by

$$
W=\int_{0}^{u^{+}}\left(1+\frac{\varepsilon_{m}}{\nu}\right) d u^{+}
$$

is introduced into Eq. (6), and one gets

$$
\frac{\partial^{2} W}{\partial x^{2}}+\frac{\partial^{2} W}{\partial y^{2}}=\frac{1 d p}{\mu u u^{*} d z}
$$

where $u^{*}$ is friction velocity and $u^{+}=u / u^{*}$. Comparing Eq. (8) with Eq. (1), one can find out that these two equations are similar and the laminar solution obtained in the previous section is applicable to the solution of $W$ in Eq. (8). Once the distribution of $W$ in an eccentric annulus is obtained, it is necessary to transform $W$ to nondimensional velocity, $u^{+}$, in the next step. For this purpose, the following assumption is made.

The relationship between the nondimensional velocity and turbulent eddy diffusivity in an eccentric annulus is just the same as that obtained in a circular tube flow.

The eddy diffusivity model proposed by van Driest $^{4)}$ and modified by Usui ${ }^{10)}$ a little later is used in this analysis.

$$
\begin{array}{ll}
\frac{\varepsilon_{m}}{\nu}=f n\left(y^{+}, R^{+}\right)^{2} D F^{2} \frac{d u^{+}}{d y^{+}} & \text {for } y^{+}<y_{c}^{+} \\
\frac{\varepsilon_{m}}{\nu}=0.07 R^{+} & \text {for } y^{+}>y_{c}^{+}
\end{array}
$$

where

$$
f n\left(y^{+}, R^{+}\right)=0.4 y^{+}-0.44 \frac{y^{+^{2}}}{R^{+}}+0.24 \frac{y^{+^{3}}}{R^{+2}}-0.06 \frac{y^{+^{4}}}{R^{+^{3}}}
$$

$$
D F=1-\exp \left(-y^{+} / 26\right)
$$

and $y_{b}^{+}$is defined as the position where eddy diffusivity becomes larger than the value of $0.07 R^{+} . R^{+}$is nondimensional tube radius $\left(=R u^{*} / \nu\right)$, and in the case of an eccentric annulus, $R$ must be replaced by the hydraulic radius. The velocity distribution is calculated by

$$
u^{+}=\int_{0}^{y^{+}} \frac{2\left(1-y^{+} / R^{+}\right) d y^{+}}{1+\sqrt{1+4 f n\left(y^{+}, R^{+}\right)^{2} D F^{2}\left(1-y^{+} / R^{+}\right)}}
$$

The relationship between $W, u^{+}$and $\varepsilon_{m} / \nu$ calculated from Eqs. (9)-(12) at $R e=10^{3}$ is shown in Fig. 2. The transformation of $W$ to $u^{+}$is easily done with this diagram. The velocity distribution obtained in this way is integrated over the cross-sectional area of an eccentric annulus to get the average velocity and friction factor. A grid structure of $50 \times 20$ mesh in $(\eta, \xi)$ bipolar coordinates was employed to carry out the computations.

At the initial stage of computation, $u^{*}$ in Eq. (8) is not known. So the value of $u^{*}$ is assumed and the above-mentioned calculation is performed to give the friction factor. Then friction velocity is estimated by $u^{*}=\sqrt{f / 2} \bar{u}$ and compared with the initial value. A trial routine is continued to give good accuracy of convergence.

\section{3 Local wall shear stress}

The local shear stress may be evaluated by the 
velocity gradient at the wall,

$$
\left.\tau\right|_{\text {wa11 }}= \pm\left.\mu \frac{d u}{d r}\right|_{\text {wa11 }}
$$

where $r$ is the radial coordinate measured from the center of inner or outer cylinder. The velocity gradient in the $r$ direction is expressed in terms of bipolar coordinates as

$$
\begin{aligned}
& \left.\tau\right|_{\substack{\text { inner } \\
\text { wa } 11}}=\tau_{1}=\left.\frac{\mu(1-\cosh \alpha \cos \xi)}{r_{1} \sinh \alpha \cos \theta} \frac{\partial u}{\partial \eta}\right|_{\eta=\alpha} \\
& \left.\tau\right|_{\substack{\text { outer } \\
\text { wal1 }}}=\tau_{2}=\left.\frac{-\mu(1-\cosh \beta \cos \xi)}{r_{2} \sinh \beta \cos \theta} \frac{\partial u}{\partial \eta}\right|_{\eta=\beta}
\end{aligned}
$$

The velocity gradient at the wall is evaluated by

$$
\left.\frac{\partial u}{\partial \eta}\right|_{\text {wa } 11}=\left.u\right|_{a t \eta=\beta-\tilde{o} \text { or } \alpha+\dot{\delta} / \delta}
$$

where $\delta$ is taken to be smaller than laminar sublayer thickness, typically $\delta=(\beta-\alpha) / 3000$ in this analysis. Computations of this work were carried out by FACOM M-190 installed in the Computer Center of Kyushu University.

\section{Results and Discussion}

The results of this work were obtained for the case of $R e=10000-200000, \phi=0.1-0.99$ and $\gamma=0.28-0.75$. Concerning the eccentricity parameter, $\phi$, the applicability of this method of prediction is restricted by the convergence of laminar velocity solution given by Eq. (5). It was confirmed that a reasonable solution was obtained at $\phi=0.99-0.1$. However, in the case of higher eccentricity, typically in the region of $\phi>0.9$, a special grid structure in $(\eta, \xi)$ coordinates was needed to obtain a uniform grid point in $(x, y)$ coordinates.

\subsection{Velocity distribution}

Calculated velocity distributions for eccentric annulus are shown in Fig. 3 compared with previous experimental and theoretical works. In Fig. 3(a) and 3 (b), the result of this work is compared with Jonsson and Sparrow's data ${ }^{5)}$ at $R e=35000, \phi=0.75$ and $\gamma=0.75$, while in Figs. 3(c) and 3(d), it is compared with Mizushina's $\mathrm{data}^{8)}$ at $R e=12200, \phi=0.5$ and $\gamma=0.468$. The laminarization in the narrower part of the cross-section is more significantly observed in the case of experimental results obtained by Jonsson and Sparrow (Fig. 3(b)) and Mizushina (Fig. 3(d)). Probably this was caused by the assumption made in the present analysis. It was assumed that the relationship between nondimensional velocity and turbulent eddy diffusivity in an eccentric annulus was just the same as that in a circular tube flow at the same Reynolds number. Howevr, local Reynolds number is obviously different between the narrow and wider parts of the cross-section. Thus the relationship between $u^{+}$and $W$ corresponding to local Reynolds number at the calculating position should be

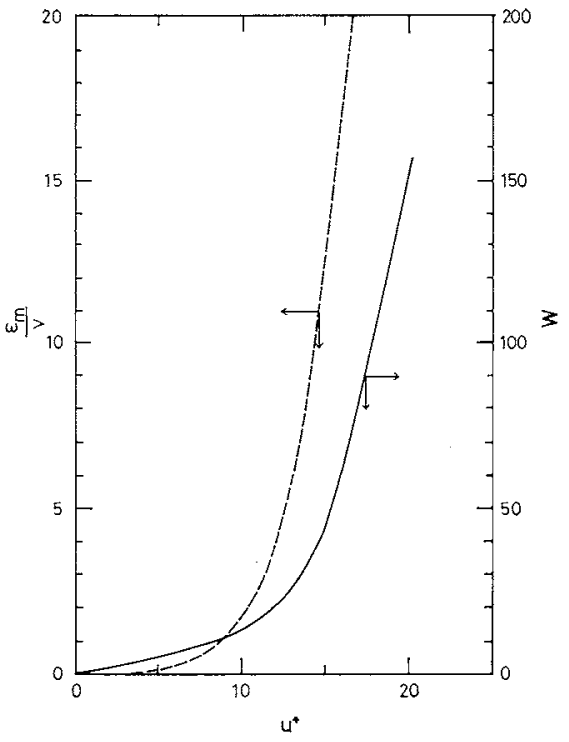

Fig. 2 Distribution of eddy diffusivity and the integral $W$ as function of dimensionless velocity

used in this prediction. Unfortunately, this idea has not been utilized at the present stage because of the complicated geometry of an eccentric annulus. In Figs. 3(e) and 3(f), the present calculation is compared with the theoretical result of Deissler and Taylor ${ }^{2)}$. A similar tendency to that described above is observed. Deissler and Taylor used the same assumption as that employed in the present analysis. That is, they assumed that the relationship between $u^{+}$and $\varepsilon_{m} / \nu$ in an eccentric annulus was just the same as that in the turbulent circular tube flow. They used the eddy difusivity model developed by Deissler ${ }^{2}$. Both Diessler's eddy diffusivity model and the van Driest-Type model, however, give essentially the same relationship between $u^{+}$and $\varepsilon_{m} / \nu$. Thus the discrepancy observed in Figs. 3(e) and 3(f) should be attributed to the difference in calculation procedure, i.e. the conformal mapping technique of Deissler and Taylor and the integral transformation method of the present analysis. Although it is very difficult to estimate quantitatively the error caused by Deissler and Taylor's method, the conformal mapping technique inevitably contains considerable error, and this seems to cause the difference in the velocity contour diagram.

\section{2 Friction factor}

The velocity distributions shown in Fig. 3 suggest that the present theory should result in higher friction factors than previous results at the same Reynolds number. A comparison of friction factor at a fixed eccentricity $(\phi=0.6)$ and a fixed radius ratio $(\gamma=0.28)$ is shown in Fig. 4. The present calculation is approximately $8.5 \%$ higher than the theory of Deissler and Taylor, and experimental results of Jonsson and Sparrow and Mizushina appear between the lines of the present prediction and that of Deissler and Taylor. 


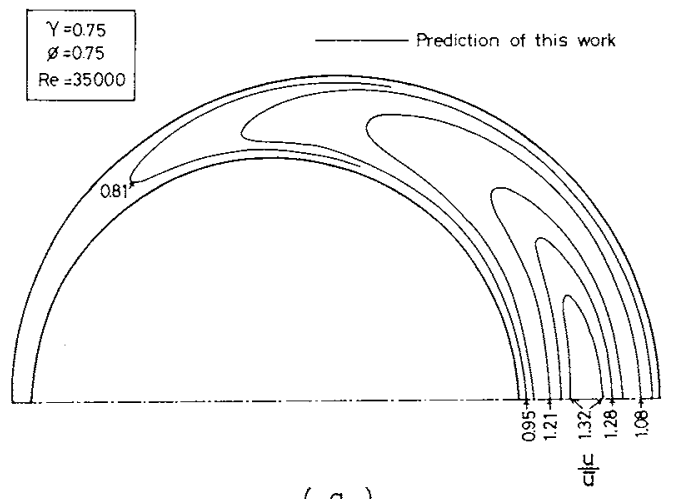

(a)

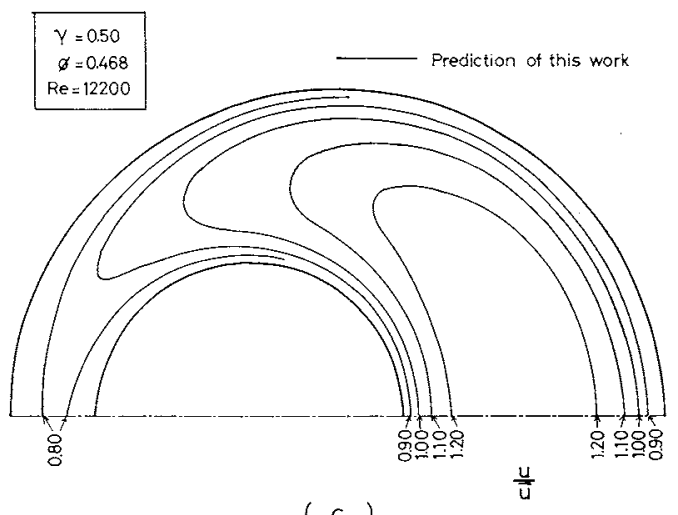

(c)

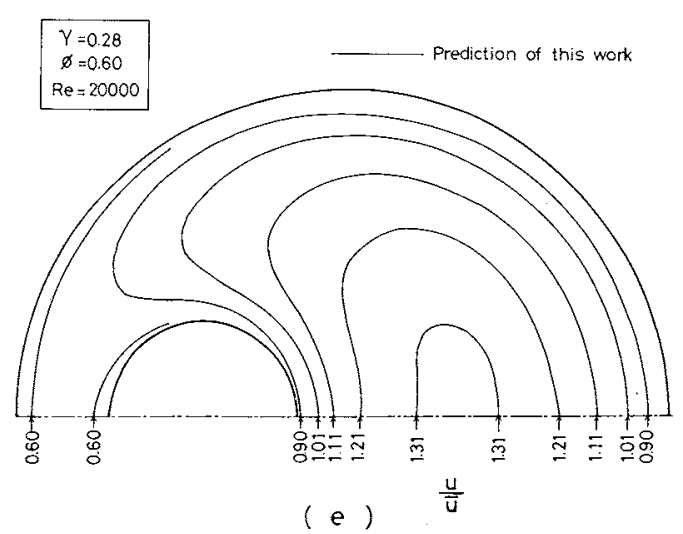

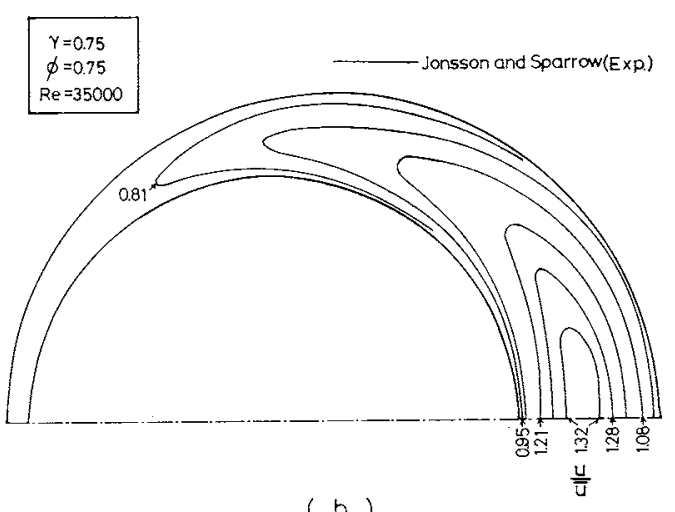

(b)

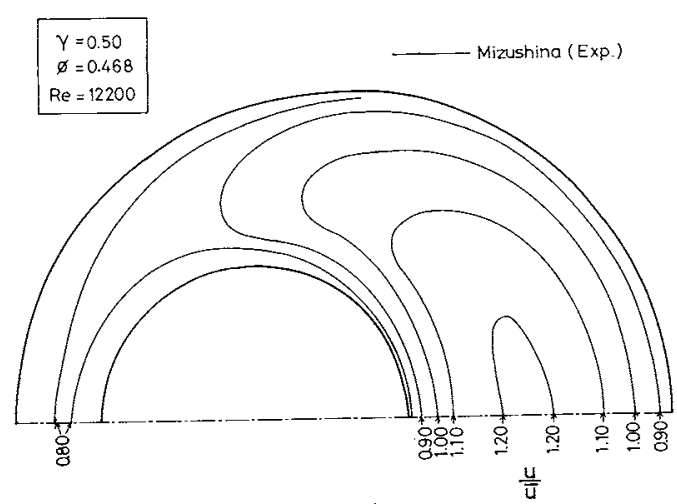

(d)

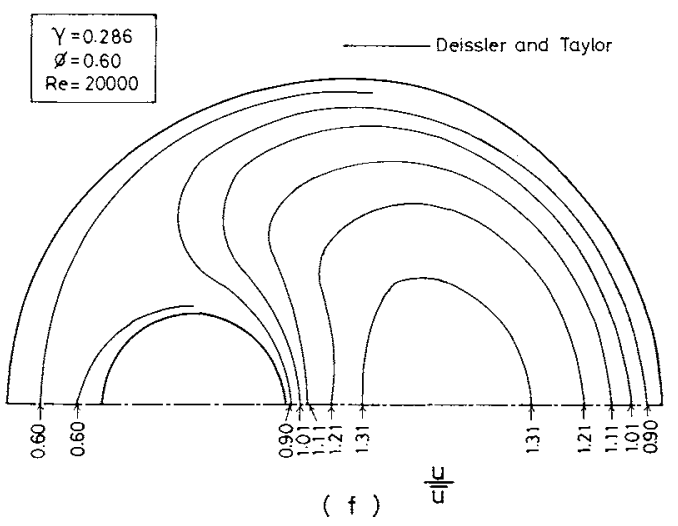

Fig. 3 Velocity contour diagrams

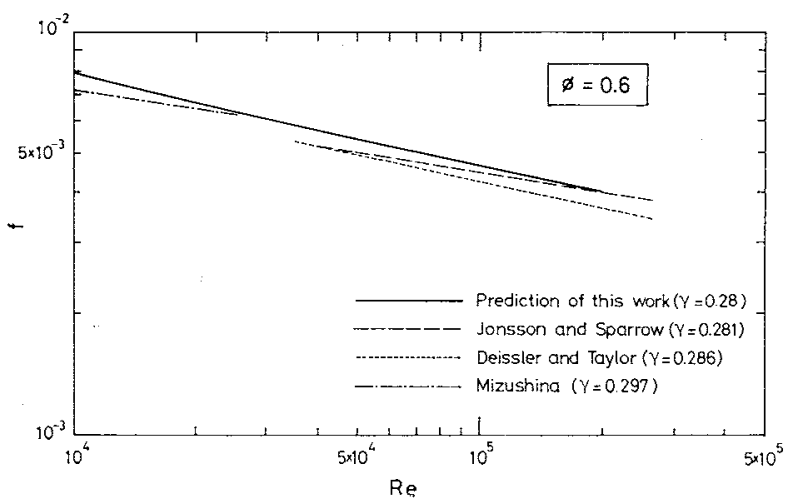

Fig. 4 Comparison of friction factor results at $\phi=0.6$ and $\gamma=0.28$

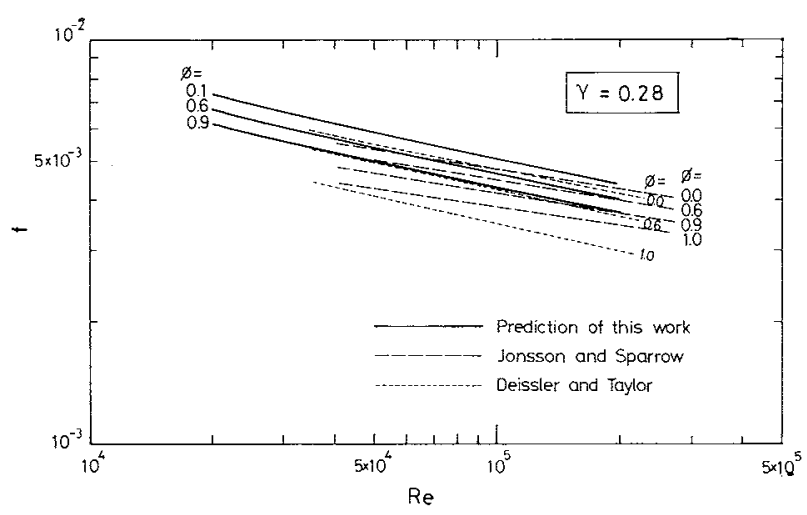

Fig. 5 Comparison of friction factor results at $r=0.28$ 
The dependence of friction factor on the eccentricity parameter is shown in Fig. 5. The friction factor decreases with increasing eccentricity. Although the agreement with Jonsson and Sparrow's experimental data is not good in the lower Reynolds number range, the present prediction can represent almost the same dependence of friction factor decrease with increase of eccentricity as the experimental results, while the prediction of Deissler and Taylor seems to overestimate the decrease of friction factor. Thus we can conclude that the present theory is effective in the prediction of friction factor of turbulent eccentric annular flow within an error less than $8 \%$ compared with the experimental results.

\subsection{Circumferential variation of wall shear stress}

The distribution of local shear stress on the bounding walls was calculated by Eq. (14). As suggested from the analysis, the present calculation gives essentially a profile of local shear stress similar to that obtained under laminar condition. It was confirmed that the relative local shear stress distribution, $\tau_{1} / \bar{\tau}_{1}$ or $\tau_{2} / \bar{\tau}_{2}$, of this work coincided with the results of laminar flow given by Snyder and Goldstein ${ }^{9}$ within an error of $2 \%$. This seems to be the main defect of the present analysis. However, it is worthwhile to discuss the difference between the analysis and the experimental results in turbulent flow.

Experimental results of Jonsson and Sparrow are compared with the present calculation in Fig. 6. Instead of the above-mentioned defect, the distribution of local shear stress is in good agreement with the experimentally determined wall shear stress. Even in the case of high eccentricity, $\phi=0.9$, the agreement between theoretical and experimental results is quite good. This means that the distribution of local shear stress in turbulent eccentric annular flow is not so different from that in laminar flow.

\section{Conclusion}

A theoretical method of prediction for the turbulent flow in an eccentric annulus was presented, and the results of calculation were compared with experimental results in the literature. Although the analysis cannot predict the secondary flow in a non-circular duct flow, the following conclusions were obtained.

1) The dependence of friction factor on eccentricity and radius ratio is well explained by the prediction of this work. Especially in the higher Reynolds number range, the predicted friction factor coincides quantitatively with the experimental results.

2) Previous experimental results of velocity distribution show more significant laminarization in the narrower part of the cross-section than does the present prediction.

3) The circumferential distribution of local wall

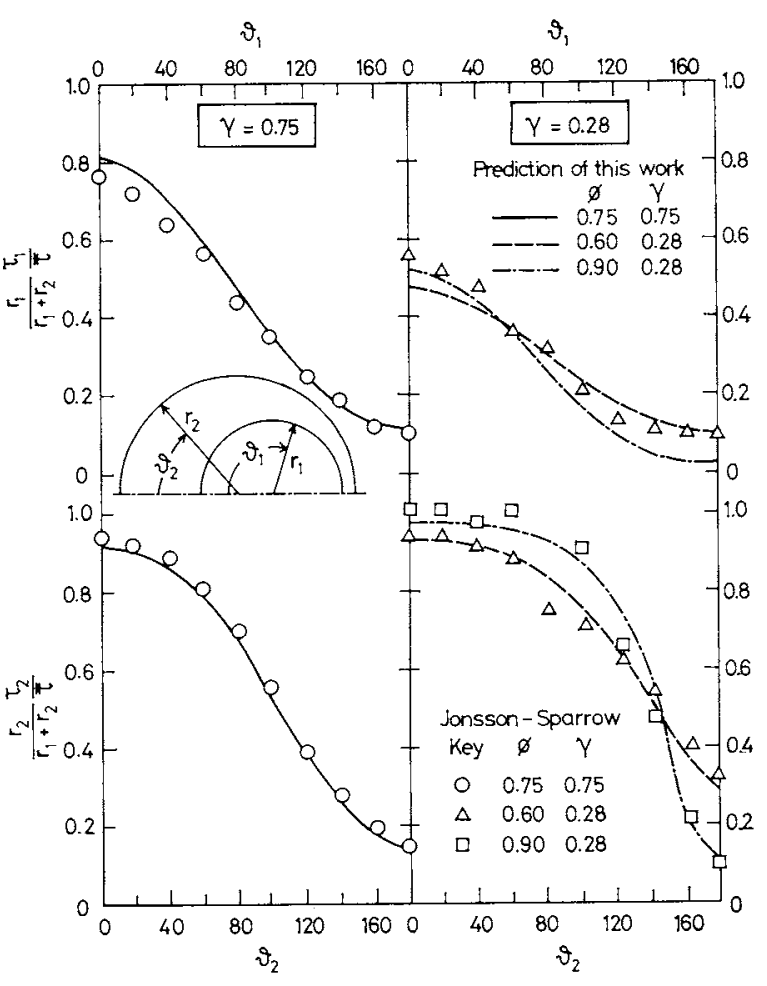

Fig. 6 Circumferential distributions of local wall shear stress

shear stress in turbulent eccentric annular flow is almost the same as that in laminar flow.

\section{Acknowledgment}

The authors would like to express their appreciation to Professor T. F. Irvine, Jr. in State Univ. New York at Stony Brook for his helpful and stimulating discussion.

$\begin{array}{llr}\text { Nomenclature } & \\ c & =\text { constant given by Eq. (3a) } & {[-]} \\ e & =\text { distance between the inner and outer } & \\ & \text { cylinders } & {[\mathrm{m}]} \\ f & =\text { friction factor } & {[-]} \\ P & =\text { static pressure } & {[\mathrm{Pa}]} \\ R & =\text { pipe radius } & {[\mathrm{m}]} \\ R e & =\text { Reynolds number } & {[-]} \\ r_{1} & =\text { radial coordinate on the inner cylinder } & {[\mathrm{m}]} \\ r_{2} & =\text { radial coordinate on the outer cylinder } & {[\mathrm{m}]} \\ u & =\text { longitudinal velocity } \\ \bar{u} & =\text { average velocity } & {[\mathrm{m} / \mathrm{s}]} \\ u^{*} & =\text { friction velocity } & {[\mathrm{m} / \mathrm{s}]} \\ u^{+} & =\text {nondimensional velocity }\left(=u / u^{*}\right) & {[\mathrm{m} / \mathrm{s}]} \\ v & =\text { dimensionless velocity defined by Eq. }(4 \mathrm{~b}) & {[-]} \\ W & =\text { integral defined by Eq. }(7) & {[-]} \\ x, y, z & =\text { space coordinates defined in Fig. 1 } & {[\mathrm{m}]} \\ y^{+} & =\text {nondimensional distance from the wall } & {[-]} \\ \alpha, \beta & = & \\ \gamma & =\text { constants given by Eqs. (3b) and }(3 \mathrm{c}) & {[-]} \\ \varepsilon_{m} & =\text { radius ratio }\left(=r_{1} / r_{2}\right) & {[-]} \\ \eta, \xi & =\text { bipolar coordinates defined by Eq. }(2) & {\left[\mathrm{m}^{2} / \mathrm{s}\right]} \\ \theta_{1} & =\text { angular coordinate on the inner cylinder } & {[-]} \\ \theta_{2} & =\text { angular coordinate on the outer cylinder } & {[\mathrm{deg}]} \\ \mu^{*} & =\text { viscosity } & {[\mathrm{Pa} \cdot \mathrm{s}]} \\ \nu & =\text { kimematic viscosity } & {\left[\mathrm{m}^{2} / \mathrm{s}\right]}\end{array}$




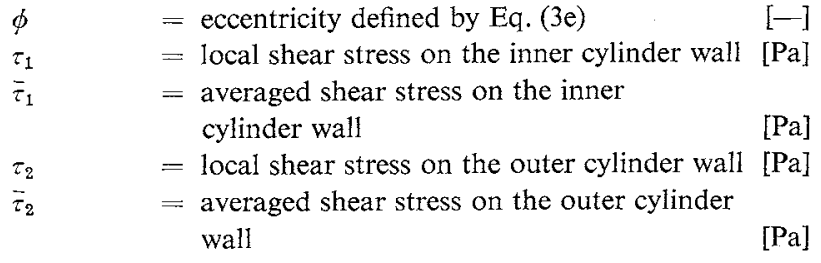

Literature Cited

1) Aly, A. M. M., A. C. Trupp and A. D. Gerrard: J. Fluid Mech., 85, 57 (1978).

2) Deissler, R. G. and M. F. Taylor: NACA Tech. Note 3451 (1955).
3) Dodge, N. A.: ASME paper No. 63-WA-11 (1963).

4) Driest, E. R. van: J. Aeronaut. Sci., 23, 1007 (1956).

5) Jonsson, V. K. and E. M. Sparrow: J. Fluid Mech., 25, 65 (1966).

6) Krajewski, B.: Int. J. Heat Mass Transfer, 13, 1819 (1970).

7) Launder, B. E. and W. M. Ying: "Fully-developed turbulent flow in ducts of square cross-section", Imperial College of Sci. \& Tech. TM-TN-A-11 (1971).

8) Mizushina, T.: Dept. Chem. Eng., Kyoto Univ., private information.

9) Snyder, O. E. and G. A. Goldstein: AIChE J., 11, 462 (1965).

10) Usui, H.: Ph. D. thesis, Kyoto Univ. (1975).

\title{
A NEW SIMULATION METHOD FOR ABSORBER, STRIPPER AND REBOILED ABSORBER WITH MULTICOMPONENT NON-IDEAL SYSTEMS
}

\author{
MOMINUDDIN CHOWDHURY, TAKESHI ISHIKAWA AND \\ Mitsuno HIRATA \\ Department of Chemical Engineering, Faculty of Engineering, \\ Tokyo Metropolitan University, Tokyo 158
}

\begin{abstract}
This rigorous and effective new simulation method is developed on the concept of a simple, stable algorithm so as to ensure fast and reliable convergence for a wide range of absorption problems. The main characteristic of this method lies in the technique of solving only two objective functions for stoichiometric and enthalpy balance equations by a matrix equation requiring the inversion, only once per problem, of a large matrix consisting of the derivatives of these functions with respect to two independent variables: temperature and vapor rate. The stability of this method is demonstrated here by its capability of solving absorption problems involving both wideand narrow-boiling mixtures, a stripper, an absorber with intermediate feed and cooling, reboiled absorber, and particularly, multicomponent absorption problems with non-ideal systems. The solutions of some of these problems predicted by this method are compared with the available results and are found to be in good agreement.
\end{abstract}

\section{Introduction}

Absorption is a countercurrent unit process in which selected components of a gas mixture are separated by using a suitable solvent. Water is the most common solvent, but heavy oil is also used for hydrocarbon absorption. This kind of separation process is governed mainly by the solubilities of the component gases in solvent and the heat effects (heat of absorption of the solute, heat absorbed or liberated by the evaporation or condensation of the solvent) that are usually encountered. Again, such a multicomponent separation process is generally expressed by an equilibrium-

Received February 28, 1980. Correspondence concerning this article should be addressed to M. Hirata. stage model involving model equations comprising material balances, equlibrium relationships, heat balances, and stoichiometric equations. These model equations become numerous and non-linear in nature. As a result, their solutions require an iterative scheme. The iterative methods developed up to now for a plate absorber are relaxation ${ }^{9)}$, single- $\theta^{7)}$, multi- $\theta^{7)}$, Sujata ${ }^{10)}$ Naphtali-Sandholm ${ }^{8)}$, Tomich ${ }^{6,11}$, Chowdhury, et al. ${ }^{4}$, etc. However, in the perspective of the published reports on these methods, it can be stated that in these methods greater emphasis is placed on the development of convergence technique than on the important matter of solving a wide range of absorption problems. As a result, they are expected to give rise to difficulties in predicting the performance of absorbers involving 